Article

\title{
On Neutrosophic Triplet Groups: Basic Properties, NT-Subgroups, and Some Notes
}

\author{
Xiaohong Zhang ${ }^{1,2, *}$ (D), Qingqing $\mathrm{Hu}^{1}$, Florentin Smarandache ${ }^{3}{ }^{(\mathbb{D})}$ and Xiaogang An ${ }^{1}$ \\ 1 School of Arts and Sciences, Shaanxi University of Science \& Technology, Xi'an 710021, China; \\ huqingqing122@163.com (Q.H.); anxiaogang@sust.edu.cn (X.A.) \\ 2 College of Arts and Sciences, Shanghai Maritime University, Shanghai 201306, China \\ 3 Department of Mathematics, University of New Mexico, Gallup, NM 87301, USA; smarand@unm.edu \\ * Correspondence: zhangxiaohong@sust.edu.cn or zhangxh@shmtu.edu.cn; Tel.: +86-189-1653-8256
}

Received: 20 June 2018; Accepted: 13 July 2018; Published: 17 July 2018

\begin{abstract}
As a new generalization of the notion of the standard group, the notion of the neutrosophic triplet group (NTG) is derived from the basic idea of the neutrosophic set and can be regarded as a mathematical structure describing generalized symmetry. In this paper, the properties and structural features of NTG are studied in depth by using theoretical analysis and software calculations (in fact, some important examples in the paper are calculated and verified by mathematics software, but the related programs are omitted). The main results are obtained as follows: (1) by constructing counterexamples, some mistakes in the some literatures are pointed out; (2) some new properties of NTGs are obtained, and it is proved that every element has unique neutral element in any neutrosophic triplet group; (3) the notions of NT-subgroups, strong NT-subgroups, and weak commutative neutrosophic triplet groups (WCNTGs) are introduced, the quotient structures are constructed by strong NT-subgroups, and a homomorphism theorem is proved in weak commutative neutrosophic triplet groups.
\end{abstract}

Keywords: neutrosophic triplet group (NTG); NT-subgroup; homomorphism theorem; weak commutative neutrosophic triplet group

\section{Introduction}

The importance of group theory is self-evident. It is widely used in many fields, such as physics, chemistry, engineering, and so on. It is a very good mathematical tool to describe the symmetry of nature $[1,2]$. As a more general concept, Molaei introduced the new notion of generalized group in $1999[3,4]$, and some researchers studied its properties [5,6].

The concept of neutrosophic set is introduced by F. Smarandache, it is a generalization of (intuitionistic) fuzzy sets [7]. The neutrosophic set theory is applied to algebraic structures, multiple attribute decision-making, and so on [8-13]. Recently, F. Smarandache and Mumtaz Ali in [14,15], for the first time, introduced the new notion of neutrosophic triplet group (NTG), which is another generalization of classical group. It is easy to verify that all generalized groups are neutrosophic triplet group. Note that, in this paper, the notion of neutrosophic triplet group, indeed, is the neutrosophic extended triplet group in [14].

Until now, for neutrosophic triplet group, some research articles are published [16-21]. At the same time, there are still some misunderstandings about this new algebraic structure. This paper will clarify some misunderstandings, especially pointing out some erroneous conclusions in [18] and will try to give improved results. In Section 2, we give some examples to illustrate which conclusions are incorrect and some misunderstandings have led to the emergence of these results. In Section 3, we prove some new important properties of neutrosophic triplet groups. In Section 4, we give some 
new concepts, include NT-subgroups, strong NT-subgroups, and weak commutative neutrosophic triplet groups (WCNTGs), and prove a homomorphism theorem of weak commutative neutrosophic triplet groups.

\section{Preliminaries}

As we all know, the concept of group is a useful tool to characterize symmetry. In the definition of classical group, unit element has operation invariance for any element, i.e., $e \cdot x=x \cdot e=x$ for all $x$ in a group $(G, \cdot)$, where $e$ in $G$ is the unit element. Moreover, the inverse element $x^{-1}$ of $x$ is also relative to the unit element $e$, and the inverse element is unique in the classical group. In [14,15], starting from the basic idea of neutrosophic set, a new algebraic structure, neutrosophic triplet group (briefly, NTG), is proposed. In NTG, the unit element is generalized as a neutral element, which is relative and local; that is, each element has its own neutral element; and the original inverse element concept is generalized as an anti (opposite) element, and it is relative to own neutral element, and it cannot be unique. In this way, NTG can express more general symmetry and has important theoretical and applied value.

Definition 1. Assume that $N$ is an empty set and * is a binary operation on $N$. Then, $N$ is called a neutrosophic triplet set (NTS) if for any $a \in N$, there exists a neutral of " $a$ " (denoted by neut(a)), and an opposite of " $a$ " (denoted by anti(a)) satisfying $([14,15])$ :

$$
\begin{gathered}
a^{*} \operatorname{neut}(a)=\operatorname{neut}(a)^{*} a=a ; \\
a^{*} \operatorname{anti}(a)=\operatorname{anti}(a)^{*} a=\operatorname{neut}(a) .
\end{gathered}
$$

And, the triple (a, neut(a), anti(a)) is called a neutrosophic triplet.

Note that, for a neutrosophic triplet set $(N, *), a \in N$, neut(a) and anti(a) may not be unique. In order not to cause ambiguity, we use the notations $\{$ neut $(a)\}$ and $\{$ anti $(a)\}$; they represent the sets of neut $(a)$ and $\operatorname{anti}(a)$, respectively.

Remark 1. In the original definition in [14,15], the neutral element cannot be a unit element in the usual sense, and then this restriction is removed, using the concept of a neutrosophic extended triplet by F. Smarandache [14]. That is, the classical unit element can be regarded as a special neutral element. Here, the notion of neutrosophic triplet refers to neutrosophic extended triplet.

Definition 2. Assume that $(N, *)$ is a neutrosophic triplet set. Then, $N$ is called a neutrosophic triplet group, if it satisfies $([14,15])$ :

(1) The operation * is closed, i.e., $a * b \in N, \forall a, b \in N$;

(2) The operation ${ }^{*}$ is associative, i.e., $\left(a{ }^{*} b\right){ }^{*} c=a{ }^{*}\left(b{ }^{*} c\right), \forall a, b, c \in N$

A neutrosophic triplet group $(N, *)$ is called to be commutative, if $a * b=b^{*} a, \forall a, b \in N$.

\section{Some Counterexamples and Misunderstandings on Neutrosophic Triplet Groups}

The research idea of Ref. [18] is very good, but the main results are not true. This section first gives some counterexamples, and then analyzes some of the misunderstandings on neutrosophic triplet groups.

Example 1. Denote $N=\{1,2,3,4,5\}$; the operation * on $N$ is defined by Table 1 . Then, $\left(N,{ }^{*}\right)$ is a commutative neutrosophic triplet group, and:

$$
\operatorname{neut}(1)=1,\{\operatorname{anti}(1)\}=\{1,2,3\} ; \operatorname{neut}(2)=3, \operatorname{anti}(2)=2 ; \operatorname{neut}(3)=3, \operatorname{anti}(3)=3 ;
$$


$\operatorname{neut}(4)=4,\{\operatorname{anti}(4)\}=\{1,2,3,4\} ; \operatorname{neut}(5)=4, \operatorname{anti}(5)=5$.

Table 1. Commutative neutrosophic triplet group.

\begin{tabular}{llllll}
\hline $\boldsymbol{*}$ & $\mathbf{1}$ & $\mathbf{2}$ & $\mathbf{3}$ & $\mathbf{4}$ & $\mathbf{5}$ \\
\hline $\mathbf{1}$ & 1 & 1 & 1 & 4 & 5 \\
$\mathbf{2}$ & 1 & 3 & 2 & 4 & 5 \\
$\mathbf{3}$ & 1 & 2 & 3 & 4 & 5 \\
$\mathbf{4}$ & 4 & 4 & 4 & 4 & 5 \\
$\mathbf{5}$ & 5 & 5 & 5 & 5 & 4 \\
\hline
\end{tabular}

Denote $H=\{1,2,3,4\}$, then $\left(H,{ }^{*}\right)$ is a neutrosophic triplet subgroup (according to Definition 17 in [18]). And,

$$
1 H=\{1,4\}, 2 H=\{1,2,3,4\}, 3 H=\{1,2,3,4\}, 4 H=\{4\}, 5 H=\{5\}
$$

This means that Lemma 1 (2), (4), (7), and (9) in [18] are not true:

$$
\begin{gathered}
1 \in H, \text { but } 1 H \neq H ; \\
1 H \neq 2 H \text { and } 1 H \cap 2 H \neq \varnothing ;
\end{gathered}
$$

$1 \in H$, but $1 H$ is a neutrosophic triplet subgroup (according to Definition 17 in [18]);

$$
|1 H| \neq|2 H|
$$

Moreover, $|H|=4,|N|=5$, it follows that $|H||| N \mid$; and the number of distinct aH in $N$ (according to Definition 18 in [18]) is no $|N||| H \mid$. This means that Theorem 3 in [18] are not true.

Example 2. Denote $N=\{1,2,3,4,5\}$, the operation ${ }^{*}$ on $N$ is defined by Table 2. Then, $\left(N,{ }^{*}\right)$ is a non-commutative neutrosophic triplet group, and:

$$
\begin{gathered}
\operatorname{neut}(1)=1, \operatorname{anti}(1)=1 ; \operatorname{neut}(2)=2, \operatorname{anti}(2)=2 ; \operatorname{neut}(3)=3, \operatorname{anti}(3)=3 ; \\
\operatorname{neut}(4)=4,\{\operatorname{anti}(4)\}=\{3,4\} ; \operatorname{neut}(5)=3, \operatorname{anti}(5)=5 .
\end{gathered}
$$

Table 2. Non-commutative neutrosophic triplet group.

\begin{tabular}{llllll}
\hline * & $\mathbf{1}$ & $\mathbf{2}$ & $\mathbf{3}$ & $\mathbf{4}$ & $\mathbf{5}$ \\
\hline $\mathbf{1}$ & 1 & 1 & 1 & 1 & 1 \\
$\mathbf{2}$ & 2 & 2 & 2 & 2 & 2 \\
$\mathbf{3}$ & 4 & 4 & 3 & 4 & 5 \\
$\mathbf{4}$ & 4 & 4 & 4 & 4 & 4 \\
$\mathbf{5}$ & 4 & 4 & 5 & 4 & 3 \\
\hline
\end{tabular}

Denote $H=\{1,2,3,4\}$, then $\left(H,{ }^{*}\right)$ is a neutrosophic triplet subgroup (according to Definition 17 in [18]). And:

$$
\begin{gathered}
1 H=\{1\}, H 1=\{1,2,4\} ; 2 H=\{2\}, H 2=\{1,2,4\} ; 3 H=\{3,4\}, H 3=\{1,2,3,4\} ; \\
4 H=\{4\}, H 4=\{1,2,4\} ; 5 H=\{4,5\}, H 5=\{1,2,4,5\} .
\end{gathered}
$$

It follows that Theorem 4 in [18] is not true:

$$
\operatorname{anti}(1) *(H 1) \subseteq H, \operatorname{anti}(2) *(H 2) \subseteq H, \operatorname{anti}(3)^{*}(H 3) \subseteq H, \operatorname{anti}(4) *(H 4) \subseteq H, \operatorname{anti}(5)^{*}(H 5) \subseteq H ;
$$


but $H$ is not normal (according to Definition 20 in [18]).

Moreover, anti $(5)^{*} 4=4 \in H$, thus $5={ }_{l} 4(\bmod H)$, according to Definition 19 in [18]. But $4 \neq{ }_{l} 5(\bmod H)$, this means that ${ }_{l}{ }_{l}$ is not an equivalence relation. Therefore, Proposition 2 in [18] is not true.

\section{Some New and Important Properties of Neutrosophic Triplet Groups}

As mentioned earlier, from the definition of neutrosophic triplet group, there may be multiple neutral elements neut (a) of an element $a$. We used more than a dozen personal computers, hoping to find an example to show that neutral elements of an element do not have to be unique. Unfortunately, we spent several months without finding the desired examples. This prompted us to consider another possibility: perhaps because of the associative law, every element in a neutrosophic triplet group has a unique neutral element? Recently, we succeeded to prove that this conjecture is true.

Theorem 1. Assume that $\left(N,{ }^{*}\right)$ is a neutrosophic triplet group. Then:

(1) $a \in N$, neut(a) is unique.

(2) $a \in N, \operatorname{neut}(a) * \operatorname{neut}(a)=\operatorname{neut}(a)$.

Proof. Assume $s, t \in\{$ neut $(a)\}$. Then $s^{*} a=a^{*} s=a, t^{*} a=a^{*} t=a$, and there exists $p, q$ such that:

$$
p^{*} a=a^{*} p=\mathrm{s}, q^{*} a=a^{*} q=t .
$$

Thus:

$$
\mathrm{s}^{*} t=\left(p^{*} a\right)^{*} t=p^{*}\left(a^{*} t\right)=p^{*} a=\mathrm{s} .
$$

On the other hand:

$$
\mathrm{s}^{*} t=\left(a^{*} p\right)^{*}\left(a^{*} q\right)=\left[a^{*}\left(p^{*} a\right)\right]^{*} q=\left(a^{*} \mathrm{~s}\right)^{*} q=a^{*} q=t .
$$

Therefore, $s=t=s^{*} t$. This means that neut $(a)$ is unique, and neut $(a) * \operatorname{neut}(a)=\operatorname{neut}(a)$ for any $a$ in $N$.

Remark 2. For an element a in a neutrosophic triplet group $(N, *)$, although neut (a) is unique, but we can see from Examples 1 and 2 that anti(a) is usually not unique.

Theorem 2. Let $\left(N,{ }^{*}\right)$ be a neutrosophic triplet group. Then $\forall a \in N, \forall a n t i(a) \in\{$ anti $(a)\}$,

(1) neut $(a)^{*} p=q^{*}$ neut (a), for any $p, q \in\{$ anti $(a)\}$;.

(2) neut $($ neut $(a))=$ neut $(a)$;

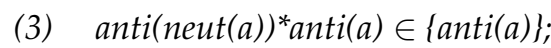

(4) neut $\left(a^{*} a\right)^{*} a=a^{*}$ neut $\left(a^{*} a\right)=a$; neut $\left(a^{*} a\right)^{*}$ neut $(a)=\operatorname{neut}(a)^{*}$ neut $\left(a^{*} a\right)=\operatorname{neut}(a)$;

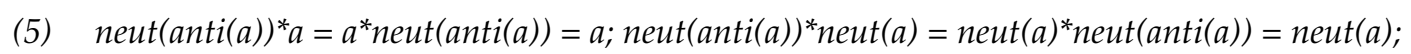

(6) $\operatorname{anti(neut}(a))^{*} a=a^{*}$ anti $($ neut $(a))=a$, for any anti(neut $\left.(a)\right) \in\{$ anti(neut $\left.(a))\right\}$;

(7) $a \in\{$ anti(neut(a)*anti(a))\};

(8) $\operatorname{neut}(a)^{*}$ anti $(a) \in\{\operatorname{anti}(a)\}$; anti(a)*neut $(a) \in\{\operatorname{anti}(a)\}$;

(9) $a \in\{$ anti(anti(a))\}, that is, there exists $p \in\{$ anti(a) $\}$ such that $a \in\{$ anti(p) $\}$;

(10) $\operatorname{neut}(a)^{*} \operatorname{anti}(\operatorname{anti}(a))=a$.

\section{Proof.}

(1) For any $p, q \in\{\operatorname{anti}(a)\}$, according the definition of neutral and opposite element, applying Theorem 1 (1), we have:

$$
\begin{aligned}
& p^{*} a=a^{*} p=\operatorname{neut}(a), q^{*} a=a^{*} q=\operatorname{neut}(a) . \\
& \operatorname{neut}(a)^{*} p=\left(q^{*} a\right)^{*} p=q^{*}\left(a^{*} p\right)=q^{*} \operatorname{neut}(a) .
\end{aligned}
$$


(2) For any anti $(a) \in\{\operatorname{anti}(a)\}$ and anti(neut $(a)) \in\{\operatorname{anti}($ neut $(a))\}$,

$\left[\operatorname{anti}(\text { neut }(a))^{*} \operatorname{anti}(a)\right]^{*} a=\operatorname{anti}(\text { neut }(a))^{*}\left[\operatorname{anti}(a)^{*} a\right]=\operatorname{anti}(\text { neut }(a))^{*}$ neut $(a)=\operatorname{neut}($ neut $(a))$.

On the other hand:

$\left\{\left[\operatorname{anti}(\text { neut }(a))^{*} \operatorname{anti}(a)\right]^{*} a\right\}^{*}$ neut $(a)=\left[\operatorname{anti}(\text { neut }(a))^{*} \operatorname{anti}(a)\right]^{*}\left[a^{*}\right.$ neut $\left.(a)\right]=$ $\left[\operatorname{anti}(\text { neut }(a))^{*} \operatorname{anti}(a)\right]^{*} a=$ neut $($ neut $(a))$.

Thus:

$$
\text { neut }(\text { neut }(a))^{*} \text { neut }(a)=\left\{\left[\operatorname{anti}(\text { neut }(a))^{*} \operatorname{anti}(a)\right]^{*} a\right\}^{*} \text { neut }(a)=\operatorname{neut}(\text { neut }(a)) \text {. }
$$

Moreover, the definition of neutral element, neut $(\text { neut }(a))^{*}$ neut $(a)=$ neut $(a)$. Therefore, neut $($ neut $(a))$ $=\operatorname{neut}(a)$.

(3) For any $\operatorname{anti}(a) \in\{\operatorname{anti}(a)\}$ and $\operatorname{anti}($ neut $(a)) \in\{\operatorname{anti}($ neut $(a))\}$, applying (2), we have:

$$
\begin{gathered}
{\left[\operatorname{anti}(\text { neut }(a))^{*} \operatorname{anti}(a)\right]^{*} a=\operatorname{anti}(\text { neut }(a))^{*}\left[\operatorname{anti}(a)^{*} a\right]=\operatorname{anti}(\text { neut }(a))^{*} \text { neut }(a)=\operatorname{neut}(\text { neut }(a))=\text { neut }(a) ;} \\
a^{*}\left[\operatorname{anti}(\text { neut }(a))^{*} \operatorname{anti}(a)\right]=\left[a^{*} \text { neut }(a)\right]^{*}\left[\operatorname{anti}(\text { neut }(a))^{*} \operatorname{anti}(a)\right]=a^{*}\left[\operatorname{neut}(a)^{*} \operatorname{anti}(\text { neut }(a))\right]^{*} \text { anti }(a)= \\
a^{*} \text { neut }(\operatorname{neut}(a))^{*} \operatorname{anti}(a)=a^{*} \text { neut }(a)^{*} \operatorname{anti}(a)=a^{*} \operatorname{anti}(a)=\operatorname{neut}(a) .
\end{gathered}
$$

Thus, anti(neut $(a))^{*}$ anti $(a) \in\{\operatorname{anti}(a)\}$.

(4) According to the definition of neutral element, using the associative law, we get:

$$
\begin{aligned}
\left(a^{*} a\right)^{*} \text { neut }\left(a^{*} a\right) & =\left(a^{*} a\right), \\
\operatorname{anti}(a)^{*}\left[\left(a^{*} a\right)^{*} \text { neut }\left(a^{*} a\right)\right] & =\operatorname{anti}(a)^{*}\left(a^{*} a\right), \\
{\left[\operatorname{anti}(a)^{*} a\right]^{*}\left[a^{*} \text { neut }\left(a^{*} a\right)\right] } & =\left[\operatorname{anti}(a)^{*} a\right]^{*} a, \\
\operatorname{neut}(a)^{*}\left[a^{*} \text { neut }\left(a^{*} a\right)\right] & =\operatorname{neut}(a)^{*} a, \\
{\left[\operatorname{neut}(a)^{*} a\right]^{*} \text { neut }\left(a^{*} a\right) } & =\operatorname{neut}(a)^{*} a, \\
a^{*} \text { neut }\left(a^{*} a\right) & =a .
\end{aligned}
$$

Similarly, we can get that neut $\left(a^{*} a\right)^{*} a=a$. Moreover:

$$
\begin{aligned}
& \operatorname{neut}(a)^{*} \text { neut }\left(a^{*} a\right)=\left[\operatorname{anti}(a)^{*} a\right]^{*} \text { neut }\left(a^{*} a\right)=\operatorname{anti}(a)^{*}\left[a^{*} \text { neut }\left(a^{*} a\right)\right]=\operatorname{anti}(a)^{*} a=\operatorname{neut}(a) . \\
& \operatorname{neut}\left(a^{*} a\right)^{*} \text { neut }(a)=\operatorname{neut}\left(a^{*} a\right)^{*}\left[a^{*} \operatorname{anti}(a)\right]=\left[\operatorname{neut}\left(a^{*} a\right)^{*} a\right]^{*} \operatorname{anti}(a)=a^{*} \operatorname{anti}(a)=\operatorname{neut}(a) .
\end{aligned}
$$

(5) For any $\operatorname{anti}(a) \in\{\operatorname{anti}(a)\}$, we have:

$$
\begin{gathered}
\operatorname{anti}(a)^{*} \text { neut }(\operatorname{anti}(a))=\operatorname{anti}(a) ; \operatorname{neut}(\operatorname{anti}(a))^{*} \operatorname{anti}(a)=\operatorname{anti}(a) . \\
a^{*}\left[\operatorname{anti}(a)^{*} \text { neut }(\operatorname{anti}(a))\right]=a^{*} \operatorname{anti}(a) ;\left[\operatorname{neut}(\operatorname{anti}(a))^{*} \operatorname{anti}(a)\right]^{*} a=\operatorname{anti}(a)^{*} a . \\
{\left[a^{*} \operatorname{anti}(a)\right]^{*} \text { neut }(\operatorname{anti}(a))=a^{*} \operatorname{anti}(a) ; \operatorname{neut}(\operatorname{anti}(a))^{*}\left[\operatorname{anti}(a)^{*} a\right]=\operatorname{anti}(a)^{*} a .} \\
\text { neut }(a)^{*} \text { neut }(\operatorname{anti}(a))=\operatorname{neut}(a) ; \operatorname{neut}(\operatorname{anti}(a))^{*} \text { neut }(a)=\operatorname{neut}(a) . \\
a^{*}\left[\text { neut }(a)^{*} \text { neut }(\operatorname{anti}(a))\right]=a^{*} \text { neut }(a) ;\left[\operatorname{neut}(\operatorname{anti}(a))^{*} \text { neut }(a)\right]^{*} a=\operatorname{neut}(a)^{*} a . \\
{\left[a^{*} \text { neut }(a)\right]^{*} \text { neut }(\operatorname{anti}(a))=a^{*} \text { neut }(a) ; \operatorname{neut}(\operatorname{anti}(a))^{*}\left[\operatorname{neut}(a)^{*} a\right]=\operatorname{neut}(a)^{*} a .} \\
a^{*} \text { neut }(\operatorname{anti}(a))=a ; \operatorname{neut}(\operatorname{anti}(a))^{*} a=a .
\end{gathered}
$$

\section{Moreover:}

$$
\text { neut }(a)^{*} \text { neut }(\operatorname{anti}(a))=\left[\operatorname{anti}(a)^{*} a\right]^{*} \text { neut }(\operatorname{anti}(a))=\operatorname{anti}(a)^{*}\left[a^{*} \text { neut }(\operatorname{anti}(a))\right]=\operatorname{anti}(a)^{*} a=\operatorname{neut}(a) \text {. }
$$$$
\text { neut }(\operatorname{anti}(a))^{*} \text { neut }(a)=\text { neut }(\operatorname{anti}(a))^{*}\left[a^{*} \text { anti }(a)\right]=\left[\text { neut }(\operatorname{anti}(a))^{*} a\right]^{*} \text { anti }(a)=a^{*} \text { anti }(a)=\text { neut }(a) \text {. }
$$

(6) For any anti(neut(a)) $\in\{$ anti(neut(a)) $\}$, by the definition of opposite element, we have:

$$
\text { neut } \left.(a)^{*} \operatorname{anti(neut}(a)\right)=\operatorname{anti}(\text { neut }(a))^{*} \text { neut }(a)=\text { neut }(\text { neut }(a)) \text {. }
$$


Applying (2), neut $($ neut $(a))=$ neut $(a)$, we get:

$$
\text { neut }(a)^{*} \operatorname{anti}(\text { neut }(a))=\operatorname{anti}(\text { neut }(a))^{*} \text { neut }(a)=\text { neut }(a) .
$$

Thus:

$$
\begin{gathered}
a^{*}\left[\text { neut }(a)^{*} \text { anti }(\text { neut }(a))\right]=a^{*} \text { neut }(a) ;\left[\operatorname{anti}(\text { neut }(a))^{*} \text { neut }(a)\right]^{*} a=\operatorname{neut}(a)^{*} a . \\
{\left[a^{*} \text { neut }(a)\right]^{*} \text { anti }(\text { neut }(a))=a^{*} \text { neut }(a) ; \operatorname{anti}(\text { neut }(a))^{*}\left[\text { neut }(a)^{*} a\right]=\operatorname{neut}(a)^{*} a .} \\
a^{*} \text { anti }(\text { neut }(a))=a ; \operatorname{anti}(\text { neut }(a))^{*} a=a .
\end{gathered}
$$

(7) For any $\operatorname{anti}(a) \in\{\operatorname{anti}(a)\}$, we have:

$$
\begin{aligned}
a^{*} \operatorname{anti}(a) & =\operatorname{anti}(a)^{*} a=\operatorname{neut}(a) . \\
{\left[a^{*} \text { neut }(a)\right]^{*} \text { anti }(a) } & =\operatorname{anti}(a)^{*}\left[\operatorname{neut}(a)^{*} a\right]=\operatorname{neut}(a) . \\
a^{*}\left[\operatorname{neut}(a)^{*} \operatorname{anti}(a)\right] & =\left[\operatorname{anti}(a)^{*} \text { neut }(a)\right]^{*} a=\operatorname{neut}(a) .
\end{aligned}
$$

Applying (1), anti $(a)^{*}$ neut $(a)=\operatorname{neut}(a)^{*} \operatorname{anti}(a)$, thus:

$$
a^{*}\left[\text { neut }(a)^{*} \operatorname{anti}(a)\right]=\left[\operatorname{neut}(a)^{*} \operatorname{anti}(a)\right]^{*} a=\operatorname{neut}(a) .
$$

Using (5), neut (a)*neut (anti (a)) =neut(a), it follows that:

$$
a^{*}\left[\text { neut }(a)^{*} \operatorname{anti}(a)\right]=\left[\operatorname{neut}(a)^{*} \operatorname{anti}(a)\right]^{*} a=\operatorname{neut}(a)^{*} \text { neut }(\operatorname{anti}(a)) \text {. }
$$

On the other hand, by (1) and Theorem 1 (2):

$$
\begin{aligned}
{\left[\text { neut }(a)^{*} \operatorname{anti}(a)\right]^{*}\left[\operatorname{neut}(a)^{*} \text { neut }(\operatorname{anti}(a))\right] } & =\operatorname{neut}(a)^{*} \text { neut }(a)^{*}\left[\operatorname{anti}(a)^{*} \text { neut }(\operatorname{anti}(a))\right]=\operatorname{neut}(a)^{*} \operatorname{anti}(a) \\
{\left[\operatorname{neut}(a)^{*} \text { neut }(\operatorname{anti}(a))\right]^{*}\left[\operatorname{neut}(a)^{*} \text { anti }(a)\right] } & =\operatorname{neut}(a)^{*}\left[\operatorname{neut}(\operatorname{anti}(a))^{*} \text { anti }(a)\right]^{*} \text { neut }(a)=\operatorname{neut}(a)^{*} \text { anti }(a) .
\end{aligned}
$$

Therefore, $a \in\left\{\operatorname{anti}\left(\right.\right.$ neut $(a)^{*}$ anti $\left.\left.(a)\right)\right\}$.

(8) Assume anti $(a) \in\{\operatorname{anti}(a)\}$, then $\left[\text { neut }(a)^{*} \text { anti }(a)\right]^{*} a=$ neut $(a)^{*}\left[\operatorname{anti}(a)^{*} a\right]=$ neut $(a)^{*}$ neut $(a)$. By Theorem $1(2), \operatorname{neut}(a)^{*}$ neut $(a)=\operatorname{neut}(a)$. Thus, $\left[\text { neut }(a)^{*} \text { anti }(a)\right]^{*} a=$ neut $(a)$. On the other hand,

$$
a^{*}\left[\text { neut }(a)^{*} \operatorname{anti}(a)\right]=\left[a^{*} \text { neut }(a)\right]^{*} \operatorname{anti}(a)=a^{*} \operatorname{anti}(a)=\operatorname{neut}(a) \text {. }
$$

Therefore:

$$
\left[\text { neut }(a)^{*} \operatorname{anti}(a)\right]^{*} a=a^{*}\left[\text { neut }(a)^{*} \operatorname{anti}(a)\right]=\operatorname{neut}(a) .
$$

This means that neut $(a)^{*}$ anti $(a) \in\{$ anti $(a)\}$. Similarly, we can get anti $(a)^{*}$ neut $(a) \in\{$ anti $(a)\}$.

(9) For any anti $(a) \in\{\operatorname{anti}(a)\}$, denote $p=\operatorname{neut}(a)^{*}$ anti(a). Using (8) we have $p \in\{\operatorname{anti}(a)\}$. Moreover, by Theorem 1 (2):

$$
\operatorname{neut}(a)^{*} \mathrm{p}=\operatorname{neut}(a)^{*}\left[\operatorname{neut}(a)^{*} \operatorname{anti}(a)\right]=\left[\operatorname{neut}(a)^{*} \operatorname{neut}(a)\right]^{*} \operatorname{anti}(a)=\operatorname{neut}(a)^{*} \operatorname{anti}(a)=\mathrm{p} .
$$

From this and applying (7), $a \in\left\{\operatorname{anti}\left(\operatorname{neut}(a)^{*} p\right)\right\}=\{\operatorname{anti}(p)\}, p \in\{\operatorname{anti}(a)\}$.

(10) Assume anti $(a) \in\{\operatorname{anti}(a)\}$ and $\operatorname{anti}(\operatorname{anti}(a)) \in\{\operatorname{anti}(\operatorname{anti}(a))\}$, by the definition of opposite element, we have:

$$
\operatorname{anti}(a)^{*} \operatorname{anti}(\operatorname{anti}(a))=\operatorname{neut}(\operatorname{anti}(a))
$$

Thus:

$$
\begin{gathered}
a^{*}\left[\operatorname{anti}(a)^{*} \operatorname{anti}(\operatorname{anti}(a))\right]=a^{*} \text { neut }(\operatorname{anti}(a)) . \\
{\left[a^{*} \operatorname{anti}(a)\right]^{*} \operatorname{anti}(\operatorname{anti}(a))=a^{*} \text { neut }(\operatorname{anti}(a)) .} \\
\text { neut }(a)^{*} \operatorname{anti}(\operatorname{anti}(a))=a^{*} \text { neut }(\operatorname{anti}(a)) .
\end{gathered}
$$


Applying (5), $a^{*}$ neut $($ anti $(a))=a$, it follows that:

$$
\operatorname{neut}(a)^{*} \operatorname{anti}(\operatorname{anti}(a))=a .
$$

Example 3. Let $Z_{6}=\{[0],[1],[2],[3],[4],[5]\}, *$ is classical mod multiplication, then $\left(Z_{6}, *\right)$ is a commutative neutrosophic triplet group, see Example 10 in [16].

We can show that (they correspond to the conclusions of Theorem 2):

(1) $[2]^{*}[4]=[5]^{*}[2],[2]^{*}[5]=[4]^{*}[2]$, that is, for any $p, q \in\{\operatorname{anti}([2])\}$, neut $([2])^{*} p=q^{*}$ neut $([2])$.

(2) $\operatorname{neut}(\operatorname{neut}([0]))=\operatorname{neut}([0])=[0], \operatorname{neut}(\operatorname{neut}([1]))=\operatorname{neut}([1])=[1], \operatorname{neut}(\operatorname{neut}([2]))=\operatorname{neut}([2])=[4]$, $\operatorname{neut}(\operatorname{neut}([3]))=\operatorname{neut}([3])=[3], \operatorname{neut}(\operatorname{neut}([4]))=\operatorname{neut}([4])=[4], \operatorname{neut}(\operatorname{neut}([5]))=\operatorname{neut}([5])=[1]$.

(3) Since neut $([2])=[4],\{\operatorname{anti}([4])\}=\{[1],[4]\}$ and $\{\operatorname{anti}([2])\}=\{[2],[5]\}$, so anti(neut $([2]))=\operatorname{anti}([4])=$ $\{[1],[4]\}$, and $[1]^{*}[2]=[2] \in\{\operatorname{anti}([2])\},[1]^{*}[5]=[5] \in\{\operatorname{anti}([5])\},[4]^{*}[2]=[2] \in\{\operatorname{anti}([2])\},[4]^{*}[5]=$ $[2] \in\{\operatorname{anti}([2])\}$. This means that anti(neut $([2])) * \operatorname{anti}([2]) \in\{\operatorname{anti}([2])\}$ for any anti $([2]) \in\{\operatorname{anti}([2])\}$ and any $\operatorname{anti}($ neut $([2])) \in\{\operatorname{anti}($ neut $([2]))\}$.

(4) $\operatorname{neut}\left([0]^{*}[0]\right)^{*}[0]=[0]^{*} \operatorname{neut}\left([0]^{*}[0]\right)=[0], \operatorname{neut}\left([0]^{*}[0]\right)^{*} \operatorname{neut}([0])=\operatorname{neut}([0])^{*}$ neut $\left([0]^{*}[0]\right)=[0]$; $\operatorname{neut}\left([1]^{*}[1]\right)^{*}[1]=[1]^{*}$ neut $\left([1]^{*}[1]\right)=[1], \operatorname{neut}\left([1]^{*}[1]\right)^{*}$ neut $([1])=\operatorname{neut}([1])^{*}$ neut $\left([1]^{*}[1]\right)=[1] ;$ and so on. This means that (4) hold for all $a \in Z_{6}$.

(5) Since $\{\operatorname{anti}([2])\}=\{[2],[5]\}$, so neut $(\operatorname{anti}([2]))=[4]$ or [1] . From $[4]^{*}[2]=[2]^{*}[4]=[2]$ and $[1]^{*}[2]=$ $[2]^{*}[1]=[2]$ we know that neut $(\operatorname{anti}([2]))^{*}[2]=[2]^{*}$ neut $(\operatorname{anti}([2]))=[2]$ for any anti $([2]) \in\{\operatorname{anti}([2])\}$ and any neut(anti([2])) $\in\{$ neut $(\operatorname{anti}([2]))\}$. Note that, since $\{$ neut $(\operatorname{anti}([2]))\}=\{[4],[1]\} ;$ when anti $([2])$ $=[5], \operatorname{neut}(\operatorname{anti}([2]))=[1] \neq \operatorname{neut}([2])$, this means that neut $(\operatorname{anti}(a))=$ neut $(a)$ is not true in general.

(6) Since $\{$ anti $($ neut $([2]))\}=\{[1],[4]\}$, from this and $[1]^{*}[2]=[2]^{*}[1]=[2]$ and $[4]^{*}[2]=[2]^{*}[4]=[2]$ we know that anti(neut $([2]))^{*}[2]=[2]^{*} \operatorname{anti}($ neut $([2]))=[2]$ for any anti(neut $\left.([2])\right) \in\{\operatorname{anti}($ neut $([2]))\}$. Note that, since $\{\operatorname{anti}($ neut $([2]))\}=\{[1],[4]\} ;$ when anti $($ neut $([2]))=[1]$, anti $($ neut $([2])) \neq \operatorname{neut}([2])$, this means that anti $($ neut $(a))=$ neut $(a)$ is not true in general.

(7) Since $\{\operatorname{anti}($ neut $([2]))\}=\{[1],[4]\}$ and $\{\operatorname{anti}([2])\}=\{[2],[5]\}$, so $\left\{\operatorname{anti}(\text { neut }([2]))^{*} \operatorname{anti}([2])\right\}=\{[2],[5]\}$, that is, [2] $\in\left\{\right.$ anti $\left.(\text { neut }([2]))^{*} \operatorname{anti}([2])\right\}$.

(8) Since neut $([2])=[4]$ and $\{\operatorname{anti}([2])\}=\left\{[2]\right.$, [5]\}, from $[4]^{*}[2]=[4]^{*}[5]=[2]$ we know that neut $([2])^{*} \operatorname{anti}([2]) \in\{\operatorname{anti}([2])\}$.

(9) Since neut $([2])=[4]$ and $\{\operatorname{anti}([2])\}=\{[2],[5]\}$, so $\{\operatorname{anti}(\operatorname{anti}([2]))\}=\{[2],[5]\}$. Thus, from $[4]^{*}[2]=[4]^{*}[5]=[2]$ we know that neut $([2])^{*} \operatorname{anti}(\operatorname{anti}([2]))=[2]$ for any anti $([2]) \in\{\operatorname{anti}([2])\}$ and $\operatorname{anti}(\operatorname{anti}([2])) \in\{\operatorname{anti}(\operatorname{anti}([2]))\}$. Note that, since $\{\operatorname{anti}(2)\}=\{[2],[5]\} ;$ when $\operatorname{anti}([2])=[5]$, $\operatorname{anti}(\operatorname{anti}([2]))=[5] \neq[2]$, this means that $\operatorname{anti}(\operatorname{anti}(a))=a$ is not true in general.

Theorem 3. Assume that $\left(N,{ }^{*}\right)$ is a commutative neutrosophic triplet group. Then $\forall a, b \in N$ :

(1) $\operatorname{neut}(a){ }^{*} \operatorname{neut}(b)=\operatorname{neut}\left(a^{*} b\right)$.

(2) $\operatorname{anti}(a) * \operatorname{anti}(b) \in\left\{\operatorname{anti}\left(a^{*} b\right)\right\}$.

Proof. If $a, b \in N$, then:

$$
\begin{gathered}
{\left[\operatorname{neut}(a)^{*} \text { neut }(b)\right]^{*}\left(a^{*} b\right)=\left\{\left[\text { neut }(a)^{*} \text { neut }(b)\right]^{*} a\right\}^{*} b=\left\{\left[\text { neut }(a)^{*} a\right]^{*} \text { neut }(b)\right\}^{*} b=\left[a^{*} \text { neut }(b)\right]^{*} b} \\
=a^{*}\left[\text { neut }(b)^{*} b\right]=a^{*} b .
\end{gathered}
$$

Similarly, we have $\left(a^{*} b\right)^{*}\left[\right.$ neut $(a)^{*}$ neut $\left.(b)\right]=a^{*} b$. That is:

$$
\left(a^{*} b\right)^{*}\left[\operatorname{neut}(a)^{*} \text { neut }(b)\right]=\left[\operatorname{neut}(a)^{*} \text { neut }(b)\right]^{*}\left(a^{*} b\right)=a^{*} b .
$$


Moreover, for any $\operatorname{anti}(a) \in\{\operatorname{anti}(a)\}$ and $\operatorname{anti}(b) \in\{\operatorname{anti}(b)\}$, we have:

$$
\begin{gathered}
{\left[\operatorname{anti}(a)^{*} \operatorname{anti}(b)\right]^{*}\left(a^{*} b\right)=\left\{\left[\operatorname{anti}(a)^{*} \operatorname{anti}(b)\right]^{*} a\right\}^{*} b=\left\{\left[\operatorname{anti}(a)^{*} a\right]^{*} \operatorname{anti}(b)\right\}^{*} b=\left[\operatorname{neut}(a)^{*} \operatorname{anti}(b)\right]^{*} b} \\
=\operatorname{neut}(a)^{*}\left[\operatorname{anti}(b)^{*} b\right]=\operatorname{neut}(a)^{*} \text { neut }(b) .
\end{gathered}
$$

Similarly, we have $\left(a^{*} b\right)^{*}\left[\operatorname{anti}(a)^{*} \operatorname{anti}(b)\right]=\operatorname{neut}(a)^{*}$ neut $(b)$. That is:

$$
\left(a^{*} b\right)^{*}\left[\operatorname{anti}(a)^{*} \operatorname{anti}(b)\right]=\left[\operatorname{anti}(a)^{*} \operatorname{anti}(b)\right]^{*}\left(a^{*} b\right)=\operatorname{neut}(a)^{*} \text { neut }(b) .
$$

Combining (1) and (2), we have neut $(a)^{*}$ neut $(b) \in\left\{\right.$ neut $\left.\left(a^{*} b\right)\right\}$. From this, by Theorem 1 , we get: neut $(a)^{*}$ neut $(b)=\operatorname{neut}\left(a^{*} b\right)$. Therefore, using $(2)$, we get anti $(a)^{*}$ anti $(b) \in\left\{\operatorname{anti}\left(a^{*} b\right)\right\}$.

\section{NT-subgroups and Weak Commutative Neutrosophic Tripet Groups}

The notion of subgroup is an important basic concept for neutrosophic triplet groups, but the definitions in the existing literatures are not consistent (see $[14,15,18,20])$. In order to avoid ambiguity, this paper gives a new definition and formally named NT-subgroup. Moreover, this section will discuss an important kind of neutrosophic triplet groups, call weak commutative neutrosophic triplet group (WCNTG). We will prove some well-known properties of WCNTG and a homomorphism theorem by special NT-subgroups.

Definition 3. Assume that $\left(N,{ }^{*}\right)$ is a neutrosophic triplet group and $H$ be a nonempty subset of $N$. Then $H$ is called a NT-subgroup of $N$ if;

(1) $a^{*} b \in H$ for all $a, b \in H$;

(2) there exists anti $(a) \in\{$ anti(a) $\}$ such that anti(a) $\in H$ for all $a \in H$, where $\{a n t i(a)\}$ is the set of opposite element of a in $(N, *)$.

Proposition 1. Assume that $\left(N,{ }^{*}\right)$ is a neutrosophic triplet group. If $H$ is a NT- subgroup of $N$, then neut $(a) \in H$ for all $a \in H$, where neut (a) is the neutral element of $a$ in $(N, *)$.

Proof. For any $a \in H$, by Theorem 1 (1) we know that neut(a) is unique. Applying Definition 3, we get that there exists $\operatorname{anti}(a) \in H$ and neut $(a)=a^{*} \operatorname{anti}(a) \in H$.

Remark 3. (1) For a NT-subgroup $H$ of $N$, where $\left(N,{ }^{*}\right)$ is a neutrosophic triplet group, $a \in H$, by Definition 3 we know that not all anti(a) is in $H$; in fact, at least one can be in $H$. (2) By Proposition 1, a $\in$ H implies neut $(a) \in H$. But $H$ does not necessarily contain neut $(b)$ for all $b \in N$. For example, let $N=Z_{6}$ in Example 3 and $H=\{[0],[2],[3],[4]\}$, then $H$ is a NT-subgroup of $\left(Z_{6},{ }^{*}\right)$, and (1) [2] $\in H$ but $\{$ anti([2])\} is not a subset of $H$; (2) $\left\{\right.$ neut $\left.(a) \mid a \in N=Z_{6}\right\}=\{[0],[1],[3],[4]\}$ is not a subset of $H$.

Definition 4. Assume that $(N, *)$ is a neutrosophic triplet group. $N$ is called a weak commutative neutrosophic triplet group (briefly, WCNTG) if $a^{*}$ neut $(b)=$ neut $(b)^{*}$ a for all $a, b \in N$.

Obviously, every commutative neutrosophic triplet group is weak commutative. The following example shows that there exists non-commutative neutrosophic triplet group which is weak commutative neutrosophic triplet group.

Example 4. Put $N=\{1,2,3,4,5,6,7\}$, and define the operation * on $N$ as Table 3. Then, $(N, *)$ is a non-commutative neutrosophic triplet group, and:

$$
\begin{gathered}
\operatorname{neut}(1)=1, \operatorname{anti}(1)=1 ; \operatorname{neut}(2)=1, \operatorname{anti}(2)=2 ; \operatorname{neut}(3)=1, \operatorname{anti}(3)=3 ; \operatorname{neut}(4)=1, \operatorname{anti}(4)=4 ; \\
\operatorname{neut}(5)=1, \operatorname{anti}(5)=6 ; \operatorname{neut}(6)=1, \operatorname{anti}(6)=5 ; \operatorname{neut}(7)=7,\{\operatorname{anti}(7)\}=\{1,2,3,4,5,6,7\}
\end{gathered}
$$


It is easy to verify that $\left(N,{ }^{*}\right)$ is a weak commutative neutrosophic triplet group.

Table 3. Weak commutative neutrosophic triplet group.

\begin{tabular}{llllllll}
\hline $\boldsymbol{*}$ & $\mathbf{1}$ & $\mathbf{2}$ & $\mathbf{3}$ & $\mathbf{4}$ & $\mathbf{5}$ & $\mathbf{6}$ & $\mathbf{7}$ \\
\hline $\mathbf{1}$ & 1 & 2 & 3 & 4 & 5 & 6 & 7 \\
$\mathbf{2}$ & 2 & 1 & 6 & 5 & 4 & 3 & 7 \\
$\mathbf{3}$ & 3 & 5 & 1 & 6 & 2 & 4 & 7 \\
$\mathbf{4}$ & 4 & 6 & 5 & 1 & 3 & 2 & 7 \\
$\mathbf{5}$ & 5 & 3 & 4 & 2 & 6 & 1 & 7 \\
$\mathbf{6}$ & 6 & 4 & 2 & 3 & 1 & 5 & 7 \\
$\mathbf{7}$ & 7 & 7 & 7 & 7 & 7 & 7 & 7 \\
\hline
\end{tabular}

Proposition 2. Assume that $\left(N,{ }^{*}\right)$ is a neutrosophic triplet group. Then $\left(N,{ }^{*}\right)$ is weak commutative if and only if $N$ satisfies the following conditions:

(1) neut $(a)^{*}$ neut $(b)=$ neut $(b)^{*}$ neut $(a)$ for all $a, b \in N$.

(2) neut (a) $)^{*}$ neut $(b)^{*} a=a^{*}$ neut(b) for all $a, b \in N$.

Proof. If $\left(N,{ }^{*}\right)$ is a weak commutative neutrosophic triplet group, then (using Definition 4$)$ :

$$
\operatorname{neut}(a)^{*} \text { neut }(b)=\operatorname{neut}(b)^{*} \text { neut }(a), \forall a, b \in \mathrm{N} .
$$

And:

$$
\operatorname{neut}(a)^{*} \text { neut }(b)^{*} a=\operatorname{neut}(a)^{*}\left[\operatorname{neut}(b)^{*} a\right]=\operatorname{neut}(a)^{*}\left[a^{*} \text { neut }(b)\right]=\left[\operatorname{neut}(a)^{*} a\right]^{*} \text { neut }(b)=a^{*} \text { neut }(b) .
$$

Conversely, assume that $N$ satisfies the conditions (1) and (2) above. Then:

$$
a^{*} \text { neut }(b)=\left[\text { neut }(a)^{*} \text { neut }(b)\right]^{*} a=\left[\text { neut }(b)^{*} \text { neut }(a)\right]^{*} a=\operatorname{neut}(b)^{*}\left[\text { neut }(a)^{*} a\right]=\operatorname{neut}(b)^{*} a .
$$

From Definition 4 we know that $\left(N,{ }^{*}\right)$ is a weak commutative neutrosophic triplet group.

Proposition 3. Let $(N, *)$ be a weak commutative neutrosophic triplet group. Then $\forall a, b \in N$ :

(1) $\operatorname{neut}(a)^{*}$ neut $(b)=\operatorname{neut}\left(b^{*} a\right)$;

(2) $\operatorname{anti}(a)^{*} \operatorname{anti}(b) \in\left\{\operatorname{anti}\left(b^{*} a\right)\right\}$.

Proof. If $a, b \in N$, then:

$$
\begin{aligned}
{\left[\operatorname{neut}(a)^{*} \text { neut }(b)\right]^{*}\left(b^{*} a\right)=} & \{[ \\
& \text { neut } \left.\left.(a)^{*} \text { neut }(b)\right]^{*} b\right\}^{*} a=\left\{\text { neut }(a)^{*}\left[b^{*} \text { neut }(b)\right]\right\}^{*} a=\left[\text { neut }(a)^{*} b\right]^{*} a \\
& {\left[b^{*} \text { neut }(a)\right]^{*} a=b^{*}\left[\text { neut }(a)^{*} a\right]=b^{*} a . }
\end{aligned}
$$

Similarly, we have $\left(b^{*} a\right)^{*}\left[\right.$ neut $(a)^{*}$ neut $\left.(b)\right]=b^{*} a$. That is:

$$
\left(b^{*} a\right)^{*}\left[\text { neut }(a)^{*} \text { neut }(b)\right]=\left[\operatorname{neut}(a)^{*} \text { neut }(b)\right]^{*}\left(b^{*} a\right)=b^{*} a .
$$

Moreover, for any $\operatorname{anti}(a) \in\{\operatorname{anti}(a)\}$ and $\operatorname{anti}(b) \in\{\operatorname{anti}(b)\}$, we have:

$$
\begin{gathered}
{\left[\operatorname{anti}(a)^{*} \operatorname{anti}(b)\right]^{*}\left(b^{*} a\right)=\left\{\left[\operatorname{anti}(a)^{*} \operatorname{anti}(b)\right]^{*} b\right\}^{*} a=\left\{\operatorname{anti}(a)^{*}\left[\operatorname{anti}(b)^{*} b\right]\right\}^{*} a=\left[\operatorname{anti}(a)^{*} \text { neut }(b)\right]^{*} a} \\
=\operatorname{anti}(a)^{*}\left[\operatorname{neut}(b)^{*} a\right]=\operatorname{anti}(a)^{*}\left[a^{*} \text { neut }(b)\right]=\left[\operatorname{anti}(a)^{*} a\right]^{*} \text { neut }(b)=\operatorname{neut}(a)^{*} \text { neut }(b) .
\end{gathered}
$$

Similarly, we have $\left(b^{*} a\right)^{*}\left[\operatorname{anti}(a)^{*} \operatorname{anti}(b)\right]=$ neut $(a)^{*}$ neut $(b)$. That is:

$$
\left(b^{*} a\right)^{*}\left[\operatorname{anti}(a)^{*} \operatorname{anti}(b)\right]=\left[\operatorname{anti}(a)^{*} \operatorname{anti}(b)\right]^{*}\left(b^{*} a\right)=\operatorname{neut}(a)^{*} \text { neut }(b) .
$$


Combining (3) and (4), we have neut $(a)^{*}$ neut $(b) \in\left\{\right.$ neut $\left.\left(b^{*} a\right)\right\}$. From this, by Theorem 1 , we get neut $(a)^{*}$ neut $(b)=$ neut $\left(b^{*} a\right)$. Therefore, using $(4)$, we get anti $(a)^{*}$ anti $(b) \in\left\{\right.$ anti $\left.\left(b^{*} a\right)\right\}$.

Definition 5. Let $\left(N{ }^{*}\right)$ be a neutrosophic triplet group and $H$ be a NT-subgroup of $N$. Then $H$ is called a strong NT- subgroup of $N$ if:

(1) $\operatorname{neut}(a) \in H$ for all $a \in N$.

(2) if there exists anti(a) $\in\{$ anti $(a)\}$ and $p \in N$ such that anti $(a)^{*} b^{*}$ neut $(p) \in H$, then there exists anti $(b) \in$ $\left\{\right.$ anti(b)\} and $q \in N$ such that $a^{*}$ anti $(b)^{*}$ neut $(q) \in H$; and the inverse is true.

Example 5. Let $\left(N,{ }^{*}\right)$ be the neutrosophic triplet group in Example 4 and $H_{1}=\{1,7\}, H_{2}=\{1,5,6,7\}$. Then $H_{1}$ and $\mathrm{H}_{2}$ are two strong NT-subgroups of $\mathrm{N}$.

Proposition 4. Let $((N, *)$ be a group (as a special neutrosophic triplet group) and $H$ be a normal subgroup of $N$. Then $\left(\left(N,{ }^{*}\right)\right.$ is a weak commutative neutrosophic triplet group and $H$ is a strong NT-subgroup of $N$.

Proof. For group $\left(N,{ }^{*}\right)$ with identity $e, \operatorname{neut}(a)=e$ and $\operatorname{anti}(a)=a^{-1}$ for any $a \in N$.

It is easy to verify that $a^{*}$ neut $(b)=n e u t(b)^{*} a$ for all $a, b \in N$. From this, by Definition 4 we know that $(N, *)$ is a weak commutative neutrosophic triplet group.

For normal subgroup $H$, by Definition 3, $H$ is a NT-subgroup of $N$. Moreover, $H$ satisfies the condition in Definition 5 (1).

Now, assume that there exists anti $(a) \in\{\operatorname{anti}(a)\}$ and $p \in N$ such that $\operatorname{anti}(a)^{*} b^{*}$ neut $(p) \in H$, this means that $a^{-1 *} b \in H$. Denote $h=a^{-1 *} b \in H$. Then $a=b^{*} h^{-1}$. Since $H$ is a normal subgroup of $N, h^{-1} \in H$ and there exists $h_{1} \in H$ such that $b^{*} h^{-1}=h_{1}^{*} b$. Thus, $a=h_{1}^{*} b, a^{*} b^{-1}=h_{1} \in H$. That is, there exists $b^{-1}=\operatorname{anti}(b) \in\{\operatorname{anti}(b)\}$ and $a \in N$ such that $a^{*} \operatorname{anti}(b)^{*}$ neut $(a)=a^{*} b^{-1 *} e=a^{*} b^{-1}=h_{1} \in H$. Similarly, we can prove the inverse is true.

Therefore, $H$ satisfies the condition in Definition 5 (2), and $H$ is a strong NT-subgroup of $N$.

Theorem 4. Let $(N, *)$ be a weak commutative neutrosophic triplet group and H be a strong NT-subgroup of $N$. Define binary relation $\approx_{H}$ on $N$ as follows: $\forall a, b \in N$ :

$$
a \approx{ }_{H} b \text { if and only if there exists anti(a) } \in\{\text { anti }(a)\} \text { and } p \in N \text { such that anti }(a)^{*} b^{*} n e u t(p) \in H \text {. }
$$

Then:

(1) the binary relation $\approx_{H}$ is an equivalent relation on $N$;

(2) $a \approx{ }_{H} b$ implies $c^{*} a \approx_{H} c^{*} b$ for all $c \in N$;

(3) $a \approx_{H} b$ implies $a^{*} c \approx_{H} b^{*} c$ and $c^{*} a \approx_{H} c^{*} b$ for all $c \in N$;

(4) denote the equivalent class contained $a$ by $[a]_{H}$, and denote $N / H=\left\{[a]_{H} \mid a \in N\right\}$, define binary operation * on $\mathrm{N} / \mathrm{H}$ as follows: $[a]_{H}{ }^{*}[b]_{H}=\left[a^{*} b\right]_{H}, \forall a, b \in N$. We can obtain a homomorphism from $\left(\mathrm{N},{ }^{*}\right)$ to $\left(\mathrm{N} / \mathrm{H},{ }^{*}\right)$, that is, $f: N \rightarrow N / H ; f(a)=[a]_{H}$ for all $a \in N$.

\section{Proof.}

(1) For any $a \in N$, applying Theorem 1 we have:

$$
\operatorname{anti}(a)^{*} a^{*} \text { neut }(a)=\left[\operatorname{anti}(a)^{*} a\right]^{*} \text { neut }(a)=\operatorname{neut}(a)^{*} \text { neut }(a)=\operatorname{neut}(a) \in \mathrm{H} \text {. }
$$

Thus $a \approx_{H} a$. 
- If $a \approx_{H} b$, then there exists anti $(a) \in\{\operatorname{anti}(a)\}$ and $p \in N$ such that $\operatorname{anti}(a)^{*} b^{*} n e u t(p) \in H$. Denote $h=\operatorname{anti}(a)^{*} b^{*}$ neut $(p)$, then $h \in H$ and:

$$
\begin{gathered}
a^{*} h=a^{*}\left[\operatorname{anti}(a)^{*} b^{*} \text { neut }(p)\right], \\
a^{*} h=\operatorname{neut}(a)^{*} b^{*} \text { neut }(p), \\
a^{*} h=b^{*} \text { neut }(a)^{*} \text { neut }(p),(\text { by Definition } 4) \\
\operatorname{anti}(b)^{*}\left(a^{*} h\right)=\operatorname{anti}(b)^{*}\left[b^{*} \text { neut }(a)^{*} \text { neut }(p)\right], \\
{\left[\operatorname{anti}(b)^{*} a\right]^{*} h=\operatorname{neut}(b)^{*} \text { neut }(a)^{*} \text { neut }(p),} \\
\left\{\left[\operatorname{anti}(b)^{*} a\right]^{*} h\right\}^{*} \text { anti }(h)=\left[\operatorname{neut}(b)^{*} \text { neut }(a)^{*} \text { neut }(p)\right]^{*} \text { anti }(h), \\
\text { anti }(b)^{*} a^{*} \text { neut }(h)=\left[\operatorname{neut}(b)^{*} \text { neut }(a)^{*} \text { neut }(p)\right]^{*} \text { anti }(h) .
\end{gathered}
$$

Applying Definition 3 we have $\left[\text { neut }(b)^{*} \text { neut }(a)^{*} \text { neut }(p)\right]^{*}$ anti $(h) \in H$, thus anti $(b)^{*} a^{*}$ neut $(h) \in H$, this means that $b \approx_{H} a$.

- If $a \approx_{H} b$ and $b \approx_{H} c$, then there exists anti(a) $\in\{\operatorname{anti}(a)\}$, anti(a) $\in\{\operatorname{anti}(a)\}, p \in N$ and $q \in N$ such that anti $(a)^{*} b^{*}$ neut $(p) \in H, \operatorname{anti}(b)^{*} c^{*}$ neut $(q) \in H$. Denote $h_{1}=\operatorname{anti}(a)^{*} b^{*}$ neut $(p)$, $h_{2}=\operatorname{anti}(b)^{*} c^{*}$ neut $(q)$, then $h_{1} \in H, h_{2} \in H$ and:

$$
b^{*} h_{2}=b^{*}\left[\operatorname{anti}(b)^{*} c^{*} \text { neut }(q)\right]=\left[b^{*} \operatorname{anti}(b)\right]^{*}\left[c^{*} \text { neut }(q)\right]=\operatorname{neut}(b)^{*} c^{*} \text { neut }(q) .
$$

From this and $h_{1}=\operatorname{anti}(a)^{*} b^{*}$ neut $(p)$, using Definition 4 we get:

$$
\begin{aligned}
& h_{1}{ }^{*} h_{2} \\
& =\left[\operatorname{anti}(a)^{*} b^{*} \text { neut }(p)\right]^{*} h_{2} \\
& =\left[\operatorname{anti}(a)^{*} b\right]^{*}\left[\operatorname{neut}(p)^{*} h_{2}\right] \\
& =\left[\operatorname{anti}(a)^{*} b\right]^{*}\left[h_{2} * \operatorname{neut}(p)\right] \\
& =\operatorname{anti}(a)^{*}\left(b^{*} h_{2}\right)^{*} \text { neut }(p) \\
& =\operatorname{anti}(a)^{*}\left[\operatorname{neut}(b)^{*} c^{*} \text { neut }(q)\right]^{*} \text { neut }(p) \\
& =\operatorname{anti}(a)^{*}\left[\operatorname{neut}(b)^{*} c\right]^{*}\left[\operatorname{neut}(q)^{*} \text { neut }(p)\right] \\
& =\operatorname{anti}(a)^{*}\left[c^{*} \text { neut }(b)\right]^{*}\left[\operatorname{neut}(q)^{*} \text { neut }(p)\right] \\
& =\left[\operatorname{anti}(a)^{*} c\right]^{*}\left[\operatorname{neut}(b)^{*} \text { neut }(q)^{*} \text { neut }(p)\right]
\end{aligned}
$$

By Definition 3 we have $h_{1}{ }^{*} h_{2} \in H$; using Proposition $3(1)$, neut $(b)^{*}$ neut $(q)^{*}$ neut $(p)=$ neut $\left(p^{*} q^{*} b\right)$. Hence:

$$
\operatorname{anti}(a)^{*} c^{*} \text { neut }\left(p^{*} q^{*} b\right)=h_{1}^{*} h_{2} \in H \text {. }
$$

This means that $a \approx_{H} c$. Therefore, $\approx_{H}$ is an equivalent relation on $N$.

(2) Assume $a \approx{ }_{H} b$. Then there exists anti(a) $\in\{$ anti(a) $\}$ and $p \in N$ such that anti $(a)^{*} b^{*}$ neut $(p) \in H$. Denote: $h=\operatorname{anti}(a)^{*} b^{*}$ neut $(p)$, then $h \in H$ and:

$$
\begin{aligned}
& h^{*} \text { neut }(c) \\
& \quad=\left[\operatorname{anti}(a)^{*} b^{*} \text { neut }(p)\right]^{*} \text { neut }(c) \\
& \quad=\left[\operatorname{anti}(a)^{*} b\right]^{*}\left[\text { neut }(p)^{*} \text { neut }(c)\right] \\
& \quad=\left[\operatorname{anti}(a)^{*} b\right]^{*}\left[\text { neut }(c)^{*} \text { neut }(p)\right] \\
& \quad=\operatorname{anti}(a)^{*}\left[b^{*} \text { neut }(c)\right]^{*} \text { neut }(p) \\
& \quad=\operatorname{anti}(a)^{*}\left[\text { neut }(c)^{*} b\right]^{*} \text { neut }(p) \\
& =\left[\operatorname{anti}(a)^{*} \text { neut }(c)\right]^{*}\left[b^{*} \text { neut }(p)\right] \\
& =\left[\operatorname{anti}(a)^{*} \operatorname{anti}(c)^{*} c\right]^{*}\left[b^{*} \text { neut }(p)\right] \\
& =\left[\operatorname{anti}(a)^{*} \operatorname{anti}(c)\right]^{*}\left(c^{*} b\right)^{*} \text { neut }(p) .
\end{aligned}
$$

Using Proposition $3(2)$, anti $(a)^{*} \operatorname{anti}(c) \in\left\{\operatorname{anti}\left(c^{*} a\right)\right\}$. Thus, there exists anti $\left(c^{*} a\right) \in\left\{\operatorname{anti}\left(c^{*} a\right)\right\}$ such that:

$$
\operatorname{anti}\left(c^{*} a\right)^{*}\left(c^{*} b\right)^{*} \text { neut }(p)=h^{*} \text { neut }(c) \in H \text {. }
$$


This means that $\left(c^{*} a\right) \approx_{H}\left(c^{*} b\right)$.

(1) Assume $a \approx_{H} b$. Then there exists anti $(a) \in\{\operatorname{anti}(a)\}$ and $p \in N$ such that anti $(a)^{*} b^{*}$ neut $(p) \in H$. Applying Definition $5(2)$, there exists anti $(b) \in\{$ anti $(b)\}$ and $q \in N$ such that $a^{*}$ anti $(b)^{*}$ neut $(q) \in H$. Denote $h=a^{*} \operatorname{anti}(b)^{*}$ neut $(q)$, then $h \in H$ and:

$$
\begin{aligned}
& \operatorname{neut}(c)^{*} h \\
& =\operatorname{neut}(c)^{*}\left[a^{*} \operatorname{anti}(b)^{*} \text { neut }(q)\right] \\
& =\left[\operatorname{neut}(c)^{*} a\right]^{*}\left[\operatorname{anti}(b)^{*} \text { neut }(q)\right] \\
& =\left[a^{*} \text { neut }(c)\right]^{*}\left[\operatorname{anti}(b)^{*} \text { neut }(q)\right] \\
& =\left\{a^{*}\left[c^{*} \operatorname{anti}(c)\right]\right\}^{*}\left[\operatorname{anti}(b)^{*} \text { neut }(q)\right] \\
& =\left(a^{*} c\right)^{*}\left[\operatorname{anti}(c)^{*} \operatorname{anti}(b)\right]^{*} \text { neut }(q) .
\end{aligned}
$$

Using Proposition $3(2)$, anti $(c)^{*} \operatorname{anti}(b) \in\left\{\operatorname{anti}\left(b^{*} c\right)\right\}$. Thus, there exists $\operatorname{anti}\left(b^{*} c\right) \in\left\{\operatorname{anti}\left(b^{*} c\right)\right\}$ such that:

$$
\left(a^{*} c\right)^{*} \operatorname{anti}\left(b^{*} c\right)^{*} \text { neut }(q)=\operatorname{neut}(c)^{*} h \in H .
$$

Applying Definition 5 (2), there exists anti $\left(a^{*} c\right) \in\left\{\operatorname{anti}\left(a^{*} c\right)\right\}$ and $r \in N$ such that:

$$
\operatorname{anti}\left(a^{*} c\right)^{*}\left(b^{*} c\right)^{*} \text { neut }(r) \in H \text {. }
$$

This means that $\left(a^{*} c\right) \approx_{H}\left(b^{*} c\right)$.

(2) Using (1)-(3) we can obtain (4).

Example 6. Let $N=\{1,2,3,4,5,6,7\}$. The operation * on $N$ is defined as Table 4. Then, $(N, *)$ is a non-commutative neutrosophic triplet group, and:

$$
\begin{gathered}
\operatorname{neut}(1)=1, \operatorname{anti}(1)=1 ; \operatorname{neut}(2)=1, \operatorname{anti}(2)=2 ; \\
\operatorname{neut}(3)=1, \operatorname{anti}(3)=3 ; \operatorname{neut}(4)=1, \operatorname{anti}(4)=4 ; \\
\operatorname{neut}(5)=1, \operatorname{anti}(5)=6 ; \operatorname{neut}(6)=1, \operatorname{anti}(6)=5 ; \operatorname{neut}(7)=7, \operatorname{anti}(7)=7 .
\end{gathered}
$$

Table 4. Weak commutative neutrosophic triplet group and its strong neutrosophic triplet (NT)-subgroup.

\begin{tabular}{llllllll}
\hline $\boldsymbol{*}$ & $\mathbf{1}$ & $\mathbf{2}$ & $\mathbf{3}$ & $\mathbf{4}$ & $\mathbf{5}$ & $\mathbf{6}$ & $\mathbf{7}$ \\
\hline $\mathbf{1}$ & 1 & 2 & 3 & 4 & 5 & 6 & 1 \\
$\mathbf{2}$ & 2 & 1 & 6 & 5 & 4 & 3 & 2 \\
$\mathbf{3}$ & 3 & 5 & 1 & 6 & 2 & 4 & 3 \\
$\mathbf{4}$ & 4 & 6 & 5 & 1 & 3 & 2 & 4 \\
$\mathbf{5}$ & 5 & 3 & 4 & 2 & 6 & 1 & 5 \\
$\mathbf{6}$ & 6 & 4 & 2 & 3 & 1 & 5 & 6 \\
$\mathbf{7}$ & 1 & 2 & 3 & 4 & 5 & 6 & 7 \\
\hline
\end{tabular}

It is easy to verify that $\left(N,{ }^{*}\right)$ is a weak commutative neutrosophic triplet group. Denote $H=\{1,5$, $6,7\}$. Then $\mathrm{H}$ is a strong NT-subgroups of N.

Thus we can get that (they correspond to the conclusions of Theorem 4):

(1) The relation $\approx_{H}$ is an equivalent relation on $N$ and $N / H=\{\{1,5,6,7\},\{2,3,4\}\}$.

(2) $1 \approx_{H} 5$ implies $2^{*} 1=2 \approx_{H} 4=2 * 5$, and so on.

(3) $1 \approx_{H} 5$ implies $1 * 2=2 \approx_{H} 3=5^{*} 2$, and so on.

(4) $\left(N / H,{ }^{*}\right)=\left\{[1]_{\mathrm{H}},[2]_{\mathrm{H}}\right\},\left(N, \stackrel{*}{\simeq}(N / H, *)\right.$, where $f(1)=f(5)=f(6)=f(7)=[1]_{\mathrm{H}}$, and $f(2)=f(3)=$ $f(4)=[2]_{\mathrm{H}}$ 
Remark 4. Applying Proposition 4 we know that Theorem 4 is a generalization of homomorphism basic theorem in classical group theory. Moreover, Theorem 4 is also a generalization of related results in [17].

\section{Conclusions}

This paper studied furtherly neutrosophic triplet group (NTG) and obtained some important results. First, some examples are given to show that some results in [18] are not true. Second, some new properties of neutrosophic triplet groups are presented, in particular, the fact of unique neutral element in every neutrosophic triplet group is proved. Third, the notions of NT-subgroup and strong NT-subgroup are proposed, a special kind of NTG (called weak commutative neutrosophic triplet group) is studied, and a homomorphism theorem is presented. All these results are interesting for exploring the structure characterizations of NTG. As the next research topics, we will explore the structures of some special NTG and their relationships with related logic algebras (such as BE-algebras and pseudo-BCI algebras [21-23]).

Author Contributions: X.Z. and Q.H. proposed the original idea of the research and wrote the first draft. F.S. gave related guidance, and X.A. participated in the study.

Acknowledgments: The research was supported by National Natural Science Foundation of China (Grant No. 61573240, 61473239).

Conflicts of Interest: The authors declare no conflict of interest.

\section{References}

1. Roman, S. Fundamentals of Group Theory; Springer: New York, NY, USA, 2012; pp. 19-58.

2. Carrell, J.B. Groups, Matrices, and Vector Spaces; Springer: New York, NY, USA, 2017; pp. 11-53.

3. Molaei, M.R. Generalized groups. Bull. Inst. Polit. Di. Iase Fasc. 1999, 3, 21-24.

4. Molaei, M.R.; Tahmoresi, A. Connected topological generalized groups. Gen. Math. 2004, 12, 13-22.

5. Akinmoyewa, J.T. A study of some properties of generalized groups. Octogon Math. Mag. 2009, 17, 599-626.

6. Adeniran, J.O.; Akinmoyewa, J.T.; Solarin, A.R.T.; Jaiyeola, T.G. On some algebraic properties of generalized groups. Acta Math. Acad. Paedagog. Nyhazi. 2011, 27, 23-30.

7. Smarandache, F. Neutrosophic set-A generialization of the intuituionistics fuzzy sets. Int. J. Pure Appl. Math. 2005, 3, 287-297.

8. Song, S.Z.; Khan, M.; Smarandache, F.; Jun, Y.B. Interval neutrosophic sets applied to ideals in BCK/BCI-algebras. Neutrosophic Sets Syst. 2017, 18, 16-26.

9. Saeid, A.B.; Jun, Y.B. Neutrosophic subalgebras of BCK/BCI-algebras based on neutrosophic points. Ann. Fuzz. Math. Infor. 2017, 14, 87-97.

10. Ye, J. Projection and bidirectional projection measures of single-valued neutrosophic sets and their decision-making method for mechanical design schemes. J. Exp. Theor. Artif. Intell. 2017, 29, 1-10. [CrossRef]

11. Liu, P.; Liu, X. The neutrosophic number generalized weighted power averaging operator and its application in multiple attribute group decision making. Int. J. Mach. Learn. Cybern. 2018, 9, 347-358. [CrossRef]

12. Zhang, X.H.; Bo, C.X.; Smarandache, F.; Dai, J.H. New inclusion relation of neutrosophic sets with applications and related lattice structure. Int. J. Mach. Learn. Cybern. 2018. [CrossRef]

13. Zhang, X.H.; Bo, C.X.; Smarandache, F.; Park, C. New operations of totally dependent-neutrosophic sets and totally dependent-neutrosophic soft sets. Symmetry 2018, 10, 187. [CrossRef]

14. Smarandache, F. Neutrosophic Perspectives: Triplets, Duplets, Multisets, Hybrid Operators, Modal Logic, Hedge Algebras, and Applications; Pons Publishing House: Brussels, Belgium, 2017.

15. Smarandache, F.; Ali, M. Neutrosophic triplet group. Neural Comput. Appl. 2018, 29, 595-601. [CrossRef]

16. Zhang, X.H.; Smarandache, F.; Liang, X.L. Neutrosophic duplet semi-group and cancellable neutrosophic triplet groups. Symmetry 2017, 9, 275. [CrossRef]

17. Zhang, X.H.; Smarandache, F.; Ali, M.; Liang, X.L. Commutative neutrosophic triplet group and neutro-homomorphism basic theorem. Ital. J. Pure Appl. Math. 2017, in press.

18. Bal, M.; Shalla, M.M.; Olgun, N. Neutrosophic triplet cosets and quotient groups. Symmetry 2018, 10, 126. [CrossRef] 
19. Jaiyeola, T.G.; Smarandache, F. Inverse properties in neutrosophic triplet loop and their application to cryptography. Algorithms 2018, 11, 32. [CrossRef]

20. Jaiyeola, T.G.; Smarandache, F. Some results on neutrosophic triplet group and their applications. Symmetry 2018, 10, 202. [CrossRef]

21. Zhang, X.H.; Wu, X.Y.; Smarandache, F.; Hu, M.H. Left (right)-quasi neutrosophic triplet loops (groups) and generalized BE-algebras. Symmetry 2018, 0, 241. [CrossRef]

22. Zhang, X.H. Fuzzy anti-grouped filters and fuzzy normal filters in pseudo-BCI algebras. J. Intell. Fuzzy Syst. 2017, 33, 1767-1774. [CrossRef]

23. Zhang, X.H.; Park, C.; Wu, S.P. Soft set theoretical approach to pseudo-BCI algebras. J. Intell. Fuzzy Syst. 2018, 34, 559-568. [CrossRef]

(C) 2018 by the authors. Licensee MDPI, Basel, Switzerland. This article is an open access article distributed under the terms and conditions of the Creative Commons Attribution (CC BY) license (http:/ / creativecommons.org/licenses/by/4.0/). 